Special Issue of the 8th International Advances in Applied Physics and Materials Science Congress (APMAS 2018)

\title{
A Meshless Method Based on Symmetric RBF Collocation for Neutron Diffusion Problems
}

\author{
T. TANBAY ${ }^{a, *}$ And B. OzGEneR ${ }^{b}$ \\ ${ }^{a}$ Bursa Technical University, Department of Mechanical Engineering, 16330 Bursa, Turkey \\ ${ }^{b}$ Istanbul Technical University, Energy Institute, 34469 Istanbul, Turkey
}

\begin{abstract}
In this study we have worked on the numerical solution of the multigroup neutron diffusion equation with the symmetric radial basis function collocation method. For the spatial approximation of the neutron flux, multiquadric, inverse multiquadric, and Gaussian basis functions are used as the interpolation functions. To test the performance of the method, both external and fission source problems are considered in two-dimensional Cartesian geometry. The effect of the shape parameter on the convergence and stability of the numerical algorithm is also investigated. The results have shown that, when the multiquadric is chosen, the symmetric RBF collocation method converges exponentially, and it is possible to obtain highly accurate multiplication factors and neutron flux distributions with this algorithm.
\end{abstract}

DOI: 10.12693/APhysPolA.135.661

PACS/topics: neutron diffusion, meshless methods, radial basis functions, symmetric, collocation

\section{Introduction}

Since their introduction in 1970s, meshless methods have become a valuable tool for the numerical solution of partial differential equations (PDEs). The most significant feature of meshless methods is that the field variable of the PDE can be approximated via nodes, which are not connected in a predefined manner. The radial basis function (RBF) collocation method is a strong-form meshless approach. The method was proposed by Kansa [1] to study fluid dynamics problems. Kansa's method is also known as the asymmetric collocation method due to its asymmetric collocation matrix. The characteristics of the asymmetric RBF collocation method for the numerical solution of neutron diffusion equation is investigated in $[2,3]$.

Although cases are very rare, the asymmetric RBF collocation method may lead to a singular matrix [4]. Based on the Hermite-Birkhoff interpolation Fasshauer formulated a symmetric version of the RBF collocation method [5]. By using the derivatives of the basis functions in accordance with the differential operators of the PDE and boundary conditions, the symmetric RBF collocation yields a symmetric collocation matrix, and thus it guarantees a nonsingular algorithm. In this work, the meshless symmetric RBF collocation method is implemented for the numerical solution of the neutron diffusion equation. Multiquadric (MQ), inverse multiquadric (IMQ) and Gaussian (GA) functions are used for the spatial approximation, and the performance of the method is investigated via numerical experiments.

*corresponding author; e-mail: tayfun.tanbay@btu.edu.tr

\section{Numerical formulation}

For 2D Cartesian geometry the multigroup neutron diffusion equation with vacuum (Dirichlet) and reflective (Neumann) boundary conditions is given in operator form by

$$
\begin{array}{ll}
L \phi_{g}^{(m)}(\boldsymbol{x})=s_{g}, & \boldsymbol{x} \in \Omega, \\
B_{V} \phi_{g}(\boldsymbol{x})=0, & \boldsymbol{x} \in \Gamma_{V}, \\
B_{R} \phi_{g}(\boldsymbol{x})=0, & \boldsymbol{x} \in \Gamma_{R},
\end{array}
$$

where $\Omega$ is the inner domain, $\Gamma_{V}$ and $\Gamma_{R}$ are the vacuum and reflective boundaries, respectively, and

$$
\begin{aligned}
& L=-D_{g} \nabla^{2}+\Sigma_{r, g}-\sum_{g^{\prime}=1}^{g-1} \Sigma_{s, g^{\prime} \rightarrow g}, \\
& B_{V}=1, \quad B_{R}=\frac{\partial}{\partial n}, \\
& s_{g} \equiv \begin{cases}\frac{1}{k^{(m-1)}} \chi_{g} F^{(m-1)}, & \text { multiplying medium } \\
S_{g, \text { ext }}, & \text { nonmultiplying medium }\end{cases}
\end{aligned}
$$

Here $n$ is the normal derivative, $g=1, \ldots, G$ is the energy group index, $m$ is the iteration index, $\phi_{g}, D_{g}, \Sigma_{r, g}, k$, and $\chi_{g}$ are the neutron flux, diffusion constant, removal cross-section, multiplication factor, and fission spectrum function, respectively. $\Sigma_{s, g^{\prime} \rightarrow g}$ is the scattering crosssection from group $g^{\prime}$ to group $g, F$ is the fission source and $S_{g, e x t}$ is an external source term. The fission source is defined by

$$
F \equiv \sum_{g^{\prime}=1}^{G} \nu_{g^{\prime}} \Sigma_{f, g^{\prime}} \phi_{g^{\prime}},
$$

where $\nu_{g^{\prime}}$ and $\Sigma_{f, g^{\prime}}$ are the number of neutrons emitted per fission and fission cross-section, respectively. If the medium is multiplying, then the solution is obtained iteratively by the fission source iteration method [3]. For a non-multiplying medium the solution can be found directly. 
Numerical approximation starts by defining the nodes $D=\left\{\boldsymbol{x}_{1}, \ldots, \boldsymbol{x}_{N_{D}}\right\}, V=\left\{\boldsymbol{x}_{N_{D}+1}, \ldots, \boldsymbol{x}_{N_{D}+N_{V}}\right\}$ and $R=\left\{\boldsymbol{x}_{N_{D}+N_{V}+1}, \ldots, \boldsymbol{x}_{N_{D}+N_{V}+N_{R}}\right\}$, where $N_{D}, N_{V}$, and $N_{R}$ are the number of nodes for the inner domain, vacuum, and reflective boundaries, respectively. Then the neutron flux is interpolated as

$$
\begin{aligned}
\phi_{g}(\boldsymbol{x}) & \cong \sum_{j=1}^{N_{D}} a_{j, g} L^{j} \psi\left(\boldsymbol{x}, \boldsymbol{x}_{j}\right)+\sum_{j=N_{D}+1}^{N_{D}+N_{V}} a_{j, g} B_{V}^{j} \psi\left(\boldsymbol{x}, \boldsymbol{x}_{j}\right) \\
+ & \sum_{j=N_{D}+N_{V}+1}^{N_{\text {tot }}} a_{j, g} B_{R}^{j} \psi\left(\boldsymbol{x}, \boldsymbol{x}_{j}\right) .
\end{aligned}
$$

Here $N_{\text {tot }}=N_{D}+N_{V}+N_{R}, a_{j, g}, j=1, \ldots, N_{\text {tot }}$ are the unknown coefficients, and the superscript $j$ means that the operators act on $\psi\left(\boldsymbol{x}, \boldsymbol{x}_{j}\right)$ as if it is a function of $\boldsymbol{x}_{j}$. The radial basis function is given by

$$
\begin{array}{ll}
\psi\left(\boldsymbol{x}, \boldsymbol{x}_{j}\right)=\left\|r^{2}+c^{2}\right\|^{q} & (\mathrm{GMQ}), \\
\psi\left(\boldsymbol{x}, \boldsymbol{x}_{j}\right)=\exp \left(-\left(r^{2} / c^{2}\right)\right) & (\mathrm{GA})
\end{array}
$$

where $r$ is the distance between nodes, $r=\left\|\boldsymbol{x}-\boldsymbol{x}_{j}\right\|, c$ is the shape parameter, and $q=1 / 2$ and $q=-1 / 2$ for MQ and IMQ, respectively. Substituting Eq. (5) into (1), and collocating at interpolation nodes gives

$$
\begin{gathered}
{\left[\begin{array}{ccc}
L L^{j} \psi\left(\boldsymbol{x}_{i}, \boldsymbol{x}_{j}\right) & L B_{V}^{j} \psi\left(\boldsymbol{x}_{i}, \boldsymbol{x}_{j}\right) & L B_{R}^{j} \psi\left(\boldsymbol{x}_{i}, \boldsymbol{x}_{j}\right) \\
B_{V} L^{j} \psi\left(\boldsymbol{x}_{i}, \boldsymbol{x}_{j}\right) & B_{V} B_{V}^{j} \psi\left(\boldsymbol{x}_{i}, \boldsymbol{x}_{j}\right) & B_{V} B_{R}^{j} \psi\left(\boldsymbol{x}_{i}, \boldsymbol{x}_{j}\right) \\
B_{R} L^{j} \psi\left(\boldsymbol{x}_{i}, \boldsymbol{x}_{j}\right) & B_{R} B_{V}^{j} \psi\left(\boldsymbol{x}_{i}, \boldsymbol{x}_{j}\right) & B_{R} B_{R}^{j} \psi\left(\boldsymbol{x}_{i}, \boldsymbol{x}_{j}\right)
\end{array}\right]} \\
\times \boldsymbol{a}_{j, g}=\left[\begin{array}{c}
s_{g}\left(x_{i}\right) \\
0 \\
0
\end{array}\right] .
\end{gathered}
$$

The numerical result can be obtained by solving Eq. (7) to yield $\boldsymbol{a}_{j, g}$.

\section{Results and discussion}

To assess the performance of the symmetric RBF collocation method for the numerical solution of the multigroup neutron diffusion equation, a one-group external source problem with a constant source and a three-group fission source problem are considered. All calculations are performed with MATHEMATICA. The dimension of the domain is chosen to be $a=25 \mathrm{~cm}$ for both problems. In case of the constant source problem the nuclear data and source term are $D=1.77764 \mathrm{~cm}$, $\Sigma_{r}=0.0143676 \mathrm{~cm}^{-1}$, and $S=1 \mathrm{n} /\left(\mathrm{cm}^{3} \mathrm{~s}\right)$, respectively, and the analytical solution of this problem is given in [3]. For the fission source case the nuclear data are given in [2] which results with a $k$ value of 0.75024 . The following error criteria are used to test the accuracy of the numerical method:

for external source

$$
\epsilon_{\mathrm{RMS}}=\sqrt{\frac{1}{N_{\mathrm{tot}}} \sum_{i=1}^{N_{\mathrm{tot}}}\left[\phi\left(\boldsymbol{x}_{i}\right)-\tilde{\phi}\left(\boldsymbol{x}_{i}\right)\right]^{2}},
$$

for fission source

$$
\epsilon_{k}=\frac{|k-\tilde{k}|}{k} \times 100
$$

where $\tilde{\phi}$ and $\tilde{k}$ are the numerical neutron flux distribution and multiplication factor, respectively.

The variation of $\epsilon_{R M S}$ and $\epsilon_{k}$ with the inverse of the node distance for the external and fission source problems is illustrated in Fig. 1, where $c=a \sqrt{0.05}$. The results show that the method converges exponentially with MQ and GA basis functions, but IMQ causes instability above $h^{-1}=25$. Also, GA converges faster and produces a higher accuracy than the MQ as the number of interpolation nodes is increased.
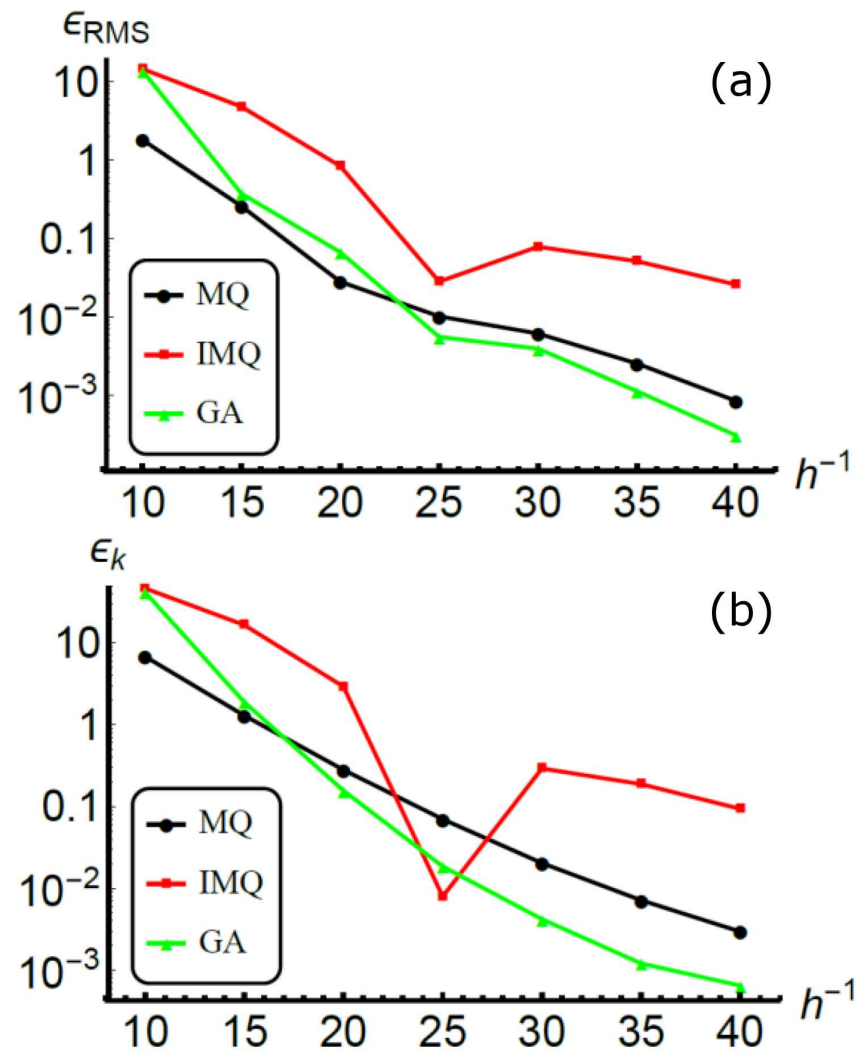

Fig. 1. Variation of $\epsilon_{\mathrm{RMS}}$ and $\epsilon_{k}$ with $h^{-1}$ for (a) external and (b) fission source problems.

The effect of the shape parameter on the accuracy of the method is presented in Fig. 2 for the two problems, where $h^{-1}=30$. In terms of stability MQ has the best performance since IMQ becomes unstable at a low value of $c$, and the GA exhibits an oscillatory behavior as the value of $c$ increases.

The above mentioned calculations are performed with uniformly distributed sets of interpolation nodes. Although the results are not presented here, random node distributions are also employed for the symmetric collocation method, and similar error behaviours are observed for both problems. 


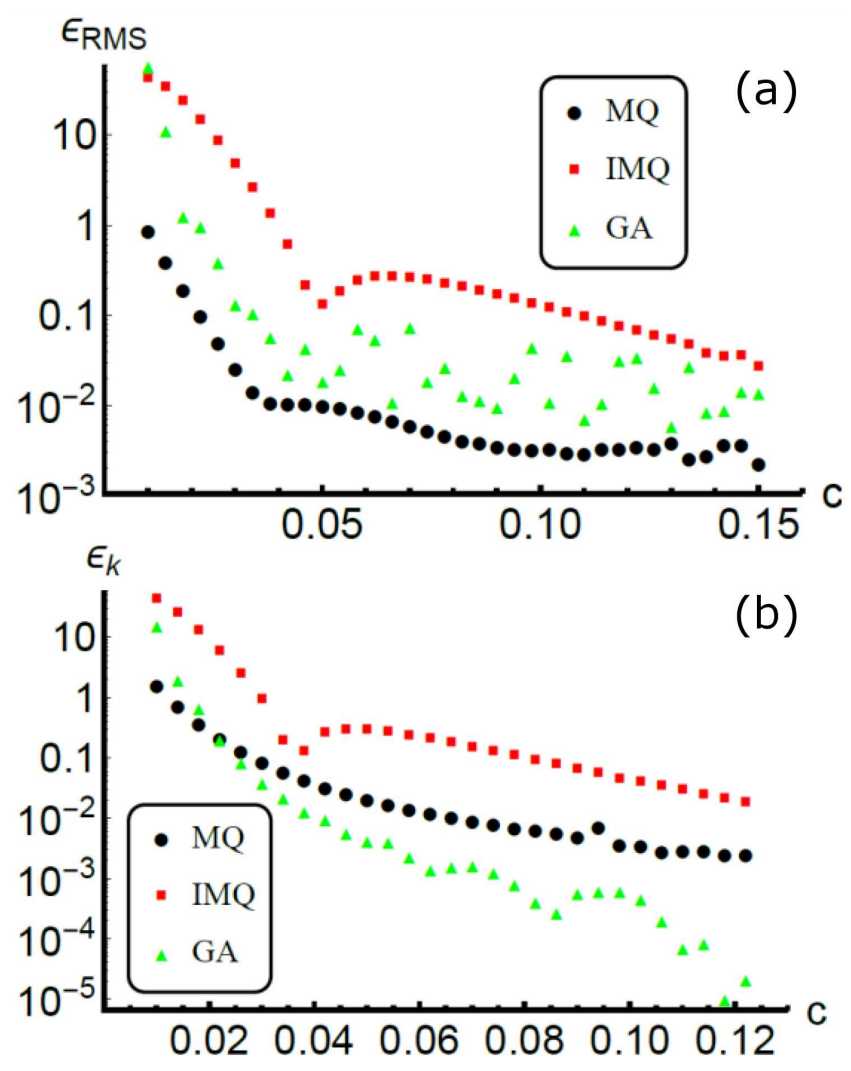

Fig. 2. Effect of the shape parameter on the accuracy for (a) external and (b) fission source problems.

\section{Conclusion}

This paper deals with the numerical solution of the neutron diffusion equation with the meshless symmetric radial basis function collocation method. Multiquadric, inverse multiquadric and Gaussian basis functions are used as interpolators for the spatial treatment. The results of the numerical tests for external and fission source problems have shown that the multiquadric is the best choice with its exponential convergence when accuracy and stability are considered together.

\section{Acknowledgments}

This work is supported by Bursa Technical University under grant no. $181 \mathrm{~N} 10$.

\section{References}

[1] E.J. Kansa, Comput. Math. Appl. 19, 127 (1990).

[2] T. Tanbay, B. Ozgener, Math. Comput. Appl. 18, 399 (2013).

[3] T. Tanbay, B. Ozgener, Eng. Anal. Bound. Elem. 46, 30 (2014).

[4] Y.C. Hon, R. Schaback, Appl. Math. Comput. 119, 177 (2001).

[5] G.E. Fasshauer, in: Surface Fitting and Multiresolution Methods, Eds. A. Le Mehaute, C. Rabut, L.L. Schumaker, Vanderbilt University Press, Nashville 1997, p. 131. 